\section{Group interpersonal psychotherapy for depression in rural Uganda: 6-month outcomes}

\author{
Randomised controlled trial
}

\section{JUDITH BASS, RICHARD NEUGEBAUER, KATHLEEN F. CLOUGHERTY, HELEN VERDELI, PRIYA WICKR AMAR ATNE, LINCOLN NDOGONI, LIESBETH SPEELMAN, MYRNA WEISSMAN and PAUL BOLTON}

\begin{abstract}
Background A randomised controlled trial comparing group interpersonal psychotherapy with treatment as usual among rural Ugandans meeting symptom and functional impairment criteria for DSM-IV major depressive disorder or sub-threshold disorder showed evidence of effectiveness immediately following the intervention.
\end{abstract}

\begin{abstract}
Aims To assess the long-term effectiveness of this therapy over a subsequent 6-month period.
\end{abstract}

Method A follow-up study of trial participants was conducted in which the primary outcomes were depression diagnosis, depressive symptoms and functional impairment.

Results At 6 months, participants receiving the group interpersonal psychotherapy had mean depression symptom and functional impairment scores respectively 14.0 points $(95 \% \mathrm{Cl}$ 12.2-15.8; $P<0.000 \mathrm{I})$ and 5.0 points (95\% Cl 3.6-6.4; $P<0.000$ I) lower than the control group. Similarly, the rate of major depression among those in the treatment arm (11.7\%) was significantly lower than that in the control arm (54.9\%) $(P<0.0001)$.

\section{Conclusions Participation in a 16 -} week group interpersonal psychotherapy intervention continued to confer a substantial mental health benefit 6 months after conclusion of the formal intervention.

Declaration of interest None.
Depression is a major cause of disability worldwide, with the World Health Organization estimating that by 2020 depressive disorders will become the leading cause of disability in low-income regions (World Health Organization, 2001). Studies in Africa have found rates ranging from $0.8 \%$ for 12 -month prevalence in Nigeria (Demyttenaere et al, 2004) to a point prevalence of $25 \%$ for those attending primary care clinics in Zimbabwe (Patel, 1998), and $15.5 \%$ and $20 \%$ for community samples in Rwanda and Uganda respectively (Bolton et al, 2002, 2004). Numerous randomised controlled trials in wealthy countries document the efficacy of psychotherapy-based interventions in reducing depressive symptoms (Jané-Llopis et al, 2003). However, psychotherapy trials in less affluent countries are sparse and have been clinic-based rather than community-based (Sumathipala et al, 2000; Araya et al, 2003; Patel et al, 2003).

This report examines the long-term effectiveness of a community-based group interpersonal psychotherapy trial conducted in rural Uganda. The study aim was to determine whether the substantial treatment benefits found immediately following the formal intervention were maintained 6 months later.

\section{METHOD}

The description of the trial and immediate post-intervention results were reported previously by Bolton et al (2003). Briefly, the trial evaluated the feasibility and effectiveness of group interpersonal psychotherapy in the treatment of depression in a population drawn from 30 randomly selected villages within Masaka and Rakai Provinces in south-west Uganda. Potential trial participants were persons identified by both key informants and themselves as suffering from at least one of two local depression-like syndromes, yo'kwekyawa and okwekubazida, loosely translated as 'self-loathing' and 'self-pity' respectively. These syndromes had been identified as similar to depression in previous qualitative research among the same population (Wilk $\&$ Bolton, 2002). To create single-gender study groups, in half the villages all the potential participants were male and in the other half, they were female. These individuals were then screened for inclusion in the trial based on responses to a depression and function assessment instrument (see below); 341 adults met eligibility criteria. Randomisation to the intervention or treatment as usual study arms was by village, so that all participants in each study village belonged to the same study arm.

For each village a list of eligible people was created and ordered according to decreasing depression severity. Working down the list, eligible individuals were invited to join the study with the goal of recruiting eight participants per village. The purpose of this procedure was to increase the likelihood of recruiting the most severely affected individuals; see Bolton et al (2003) for further description of the sampling strategy. In this way 284 individuals were invited to participate in the trial and 248 of these $(87 \%)$ consented. Ninety per cent of the 248 participants $(n=224)$ completed the trial and were assessed 2 weeks post-intervention. Of these, $96 \%$ were evaluated at the 6-month follow-up assessment (intervention group, $n=103$; control group, $n=113$ ) and are included in this report (Fig. 1).

\section{Intervention}

The intervention was conducted in singlegender groups of five to eight participants, with one group per participating village. Groups met for approximately $90 \mathrm{~min}$ once a week for 16 weeks. Each group was led by a local Ugandan of the same gender with no previous mental health or counselling experience other than training in group interpersonal psychotherapy by members of the study team (K.F.C. and H.V.). The group leaders spoke English and Luganda (the local language), had completed high school, and were available to work as counsellors for the duration of the study. Training consisted of 2 weeks of intensive instruction comprising didactic and roleplay techniques. Out of an initial 10 individuals, 9 successfully completed the counselling training. 


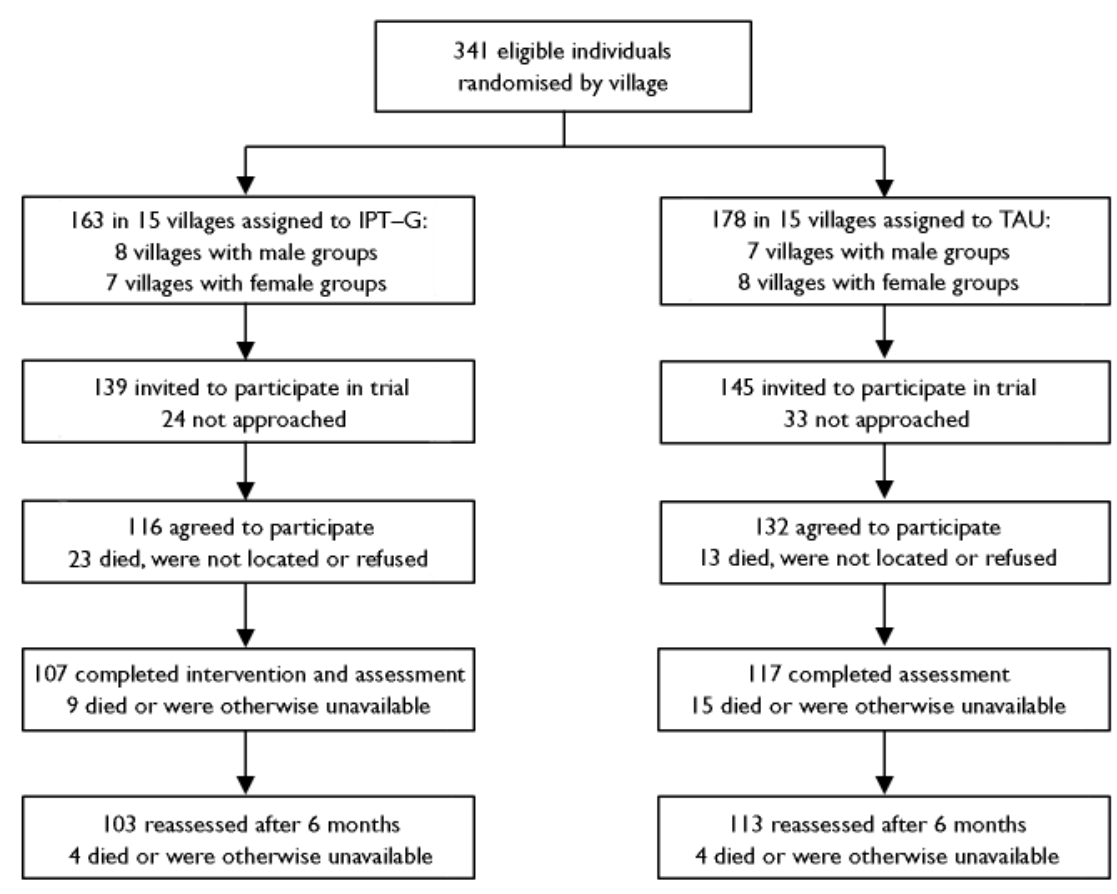

Fig. I Participation and attrition rates of individuals eligible for the study (IPT-G, group interpersonal psychotherapy; TAU, treatment as usual).

At each intervention session, the group leader encouraged each group member to review his or her current depression symptoms, to describe the prior week's events and to link both positive and negative events to his or her current mood. Group members were also encouraged to provide support and suggestions to one another. Attendance at group meetings was high, with $75 \%$ of participants present for at least $80 \%$ of the weekly sessions (13 sessions) and only $4 \%$ of participants present at fewer than 11 sessions. Reasons for absenteeism included participant illness, family illness or death, or individual work commitments. Details of the intervention and its adaptation for this population have been described by Verdeli et al (2003). In control villages, individuals did not receive any specific treatment, nor did they, in the context of this study protocol, meet in groups for some other purpose. All villagers in both study arms were free to access any locally available treatment, which may have included counselling services provided by local HIV/AIDS organisations or talking with friends, family or church members. The study protocol did not include a systematic effort to document utilisation of these locally available treatments. Prior discussions with local traditional healers indicated that they believed they were unable to treat these mental health problems effectively.

\section{Instruments}

Potential trial participants were screened using an adaptation of the depression section of the Hopkins Symptom Checklist (HSCL; Derogatis et al, 1974), an instrument widely used for research with nonWestern populations (see, for example, Mollica et al, 1987; Sabin et al, 2003).The depression sub-scale of the HSCL correlates well with other standard measures of depression and with clinical judgement of change in depression over time (Mulder et $a l, 2003)$. The instrument consisted of 15 symptoms measured using a four-point Likert scale ranging from 'not bothered at all' by the symptom (scored as 0 ) to 'bothered extremely' (scored as 3) in the previous week. Depression severity was measured as a sum of these items. To quantify functional impairment, individuals were administered a gender-specific questionnaire comprising nine items describing tasks previously identified in a qualitative study as important activities among rural Ugandans. Response options to the scale items consisted of a five-point Likert scale, ranging from having 'no more' difficulty (scored as 0) to being 'frequently unable to do the task' (scored as 4) compared with others of the same gender and age group. Individual functional impairment was measured as a sum of the responses to these items. The modification and cross-cultural validation of the HSCL with this population has been described (Bolton, 2001), as has the development and validation of the function instrument (Bolton \& Tang, 2002).

A diagnosis for major depressive disorder was generated using an algorithm originally developed by Mollica et al (1999) based on the DSM-IV A (or symptom) criteria (American Psychiatric Association, 1994). We expanded this algorithm to include the DSM-IV C and E diagnostic criteria pertaining to the composition, number and duration of symptoms and functional impairment (dysfunction) (Bolton et al, 2002). We did not obtain information for the B and D criteria (referring to measurement of criteria for a mixed episode and physiological effects of a substance or medical condition, respectively) and therefore refer to the diagnosis as a modified version of the DSM-IV diagnosis of major depressive disorder. Inclusion criteria for trial participation were being over 17 years of age; meeting all or all but one of the algorithm's DSM-IV symptom criteria for depression (the latter classification being referred to as 'sub-threshold depression'); and reporting some degree of functional impairment.

The same measures and diagnostic criteria were used for the baseline, 2-week post-intervention and 6-month follow-up assessments. In addition, questions were added to the 6-month follow-up questionnaire to assess any activities the study participants might have engaged in during the intervening 6 months with regard to their depressive symptoms. The assessments, including the HSCL and functional impairment measures, were administered in Luganda by Ugandan interviewers. Interviewers were staff members of the collaborating organisation, World Vision (http://www.worldvision.org), and had at least a high-school education, spoke English and Luganda, and were available for the duration of the study. Nine of the 10 interviewers had participated in earlier studies with the same research team. The baseline assessments were conducted in the villages, with the randomisation of village groups to intervention or control status done afterwards to ensure that interviewers were not aware of participant trial status at baseline. In an effort to keep interviewers unaware of the participants' intervention status, the post-intervention and 6-month follow-up assessments were conducted at a centrally located community 
centre. At these assessments, trial participants were transferred from their villages and were asked not to divulge either their village of origin or their treatment assignment status. To reduce measurement error that might have arisen from different interviewing styles, study participants were interviewed by the same interviewer at each stage of the study.

\section{Statistical analysis}

To test whether the initial intervention effect on depression severity and functional impairment was maintained, symptom scores both from the 2-week postintervention assessment and the 6-month follow-up assessment were compared with baseline scores. A repeated-measures analysis using a mixed model approach was conducted to allow for within-village and within-subject correlations. Separate analyses were performed of depression severity and functional impairment outcomes. The independent variables included treatment status (intervention $v$. treatment), time of assessment, a term for the interaction of treatment and time, and baseline scale scores. The interaction term tests whether the treatment effect varies by assessment period. The effect of village and participant are treated as random. Tests of significance for main effects and interaction terms were set at $P<0.05$, two-tailed using SAS version 8.0 (SAS Institute Inc., Cary, North Carolina, USA). Owing to widespread illiteracy in the region, informed consent was obtained by the interviewer reading the consent form to the participant, with consent indicated by the interviewer signing the form as a witness. This investigation was approved by the Johns Hopkins University institutional review board. In Uganda, we received approval for the study from representatives of the local government and community groups who had been involved in the previous qualitative and quantitative studies.

\section{RESULTS}

Six months after the post-intervention assessment, 103 (96\%) of the 107 participants in the intervention group who completed the trial and $113(97 \%)$ of the 117 controls were reassessed. Among those unavailable for this assessment, 6 had moved away or otherwise could not be found and 2 had died. Among the individuals available at 6 months, $52 \%$ were women and the mean (baseline) age was 46.4 years $($ s.d. $=16.0)$. The baseline mean depression scores were 23.6 (s.d.=6.5) and 24.5 (s.d.=6.2) and the functional impairment scores were 12.2 (s.d.=6.7) and 12.6 (s.d.=6.6) for the intervention and control groups respectively. There was no statistically significant difference in characteristics at baseline between participants in the two arms of the trial (Table 1). The similarity in depressive symptom means and the non-significant between-group difference in proportion of participants meeting diagnostic criteria reflect minor between-group variations in the relationship of reported symptoms to the diagnostic algorithm for major depressive disorder. The follow-up samples in each trial arm did not differ significantly from the initial baseline sample.

Mean HSCL and functional impairment scores at each assessment are presented in Table 2. The mean HSCL score of the participants receiving the intervention was about 14 points lower than that of the control group at the 2-week and 6month post-intervention assessments $(P<0.0001)$ (Table 3$)$. The analysis of functional impairment produced parallel results. The overall mean functional impairment score was about 4 points lower in the intervention group compared with the control group 2 weeks after the intervention and about 5 points lower 6 months after the intervention $(P<0.0001)$. All analyses were adjusted for time of assessment and baseline function score. In the formal repeated-measures analysis the interaction between assessment time and treatment status was not significant for the HSCL score $(P=0.71)$, indicating that the difference achieved in depression between the intervention and control groups 2 weeks after the intervention had not changed at the 6-month follow-up. The main effect of treatment allocation across both followup points was large, and significant in favour of the intervention.

At baseline $86 \%$ of participants in the intervention group met the modified diagnostic criteria for major depressive disorder and $94 \%$ of those in the control group met these criteria (prevalence difference was not statistically significant). The corresponding percentages for the 2-week postintervention assessment were $7 \%$ and $57 \%$ and at the 6-month follow-up were $12 \%$ and $55 \%$ respectively. The differences in prevalence between the study arms were statistically significant at both follow-up assessments $(P<0.0001)$.

Participants reported that during the 6 months following termination of the formal intervention 14 of the 15 therapy groups continued to meet without group leaders, with $85 \%(n=88)$ of the 103 participants who were reassessed at the 6-month follow-up attending. Figure 2 presents the mean HSCL scores for the control and intervention groups, with participants in the latter group stratified as to whether or not they reported attending any meeting with their group during the 6-month postintervention period. The difference in mean HSCL scores between those who reported attending group meetings and those not attending was not statistically significant at the 2-week post-intervention assessment, but was statistically significant $(P<0.05)$ at the 6-month follow-up.

\section{DISCUSSION}

The results of our study indicate that the marked reductions in depressive symptoms and accompanying dysfunction found in those treated with group interpersonal psychotherapy at the conclusion of the intervention were sustained 6 months later.

Table I Baseline socio-demographic and clinical characteristics of those assessed at 6-month follow-up

\begin{tabular}{lcc}
\hline Characteristic' $^{\prime}$ & Intervention group $(n=103)$ & Control group $(n=I 13)$ \\
\hline Age, years: mean (s.d.) & $47.6(15.5)$ & $45.3(16.5)$ \\
Education, years: mean (s.d.) & $4.7(2.8)$ & $3.9(3.2)$ \\
Female, $n$ (\%) & $52(50)$ & $60(53)$ \\
Depression score: mean (s.d.) & $23.6(6.5)$ & $24.5(6.2)$ \\
Function score: mean (s.d.) & $12.2(6.7)$ & $12.6(6.6)$ \\
Major depression, $n$ (\%) & $88(85)$ & $106(94)$ \\
\hline
\end{tabular}

I. Participants in the two arms of the trial did not differ significantly on any of these characteristics.

2. Major depression diagnosed using a modification of the criteria from DSM-IV (see Method). 
Table 2 Depression and function score outcomes according to trial groups and assessment period (analysis comparing trial groups as allocated with observed data only)

\begin{tabular}{lcc}
\hline & $\begin{array}{c}\text { Intervention group } \\
(n=103)\end{array}$ & $\begin{array}{c}\text { Control group } \\
(n=I I 3)\end{array}$ \\
\hline HSCL scores: mean (s.d.) & & \\
Baseline & $23.6(6.5)$ & $24.5(6.1)$ \\
2-week post-intervention & $6.1 \quad(6.3)$ & $20.6(9.0)$ \\
6-month follow-up & $6.1(7.5)$ & $20.5(10.1)$ \\
Functional impairment scores: mean (s.d.) $)^{2}$ & & \\
Baseline & $12.3(4.7)$ & $12.5(6.6)$ \\
2-week post-intervention & $4.3(4.7)$ & $8.7 \quad(7.5)$ \\
6-month follow-up & $3.6(5.4)$ & $9.5(8.1)$ \\
\hline
\end{tabular}

HSCL, Hopkins Symptom Checklist.

I. The HSCL scale consisted of 14 items, with four responses for each item related to the degree of distress due to a particular symptom (range $0-42$ points); higher scores indicate more severe depression.

2. The function scale consisted of 9 items with five responses for each item indicating degree of difficulty in completing the activity (range 0-36 points); higher scores indicate more dysfunction.

Table 3 Adjusted differences in mean score for the intervention group mean scores minus the control group mean scores

Difference in mean scores $(95 \% \mathrm{Cl})^{\prime}$

\begin{tabular}{|c|c|c|}
\hline \multicolumn{3}{|l|}{ HSCL scores } \\
\hline Baseline to 2-week follow-up & I3.9| (10.99-16.84) & $<0.0001$ \\
\hline Baseline to 6-month follow-up & $13.98(12.17-15.79)$ & $<0.0001$ \\
\hline \multicolumn{3}{|l|}{ Functional impairment scores } \\
\hline Baseline to 2-week follow-up & $4.32(2.80-5.84)$ & $<0.001$ \\
\hline Baseline to 6-month follow-up & $4.97 \quad(3.56-6.38)$ & $<0.001$ \\
\hline
\end{tabular}

HSCL, Hopkins Symptom Checklist.

I. Data refer to the difference in mean score between intervention and control groups, controlling for baseline in a random effects model.

Of note is the stability in mean depression scores in both the control group and the participants receiving interpersonal psychotherapy who reported attending any group meeting after the formal intervention was complete (Fig. 2). The stability in scores for the control group may in part be explained by the sampling strategy used in this study. Symptom severity varies with time, and members of the study sample were likely to have been selected when their symptoms were more severe. Over time without treatment, they would be expected to regress back to some population (or sample) mean reflected in the level of depressive symptoms at the 2-week and 6-month post-intervention follow-up assessments.

A similar regression to the mean would also have operated among the group receiving the intervention; however, some other factor is needed to explain why the depression scores remained stable for 6 months at levels much lower than those of the controls. One possibility is that the lingering impact of the psychotherapy was extraordinarily strong over the 6-month follow-up period. Alternatively, some effective elements of the intervention might still have been in place, as suggested by the majority of the groups continuing to meet without group leaders. Limited information is available about how often the groups met during the post-intervention period, with some respondents indicating that the groups met regularly and others describing meetings arranged more on an ad hoc basis if a group member wanted to get everyone together. Intervention participants who continued to attend meetings during the 6-month follow-up period indicated that their groups addressed a range of topics. The matter most often mentioned was development of income-generating projects for the group as a whole and for individuals within the groups. Many of the participants also noted that groups were used for informal counselling, discussing how each person was doing socially and emotionally, and advising about problems they were facing.

The $14 \%$ who responded that they did not continue to attend group meetings had higher depression scores (not statistically significant) immediately after the intervention than those who responded that they did attend meetings. However, the mean depression score for this subsample had gone up significantly at the 6-month assessment, although it remained lower than that of the control group (Fig. 2). Hence, the 6-month findings could be interpreted as evidence either of the enduring benefits of short-term treatment or of the continuing benefits of attending group meetings even in the absence of a trained group leader (or both). Assessing the relative contribution of these two factors to our study findings is not possible within the framework of the current investigation, but certainly warrants future study.

\section{Comparison with other trials}

To our knowledge, this is the first formally evaluated follow-up of a community-based randomised controlled trial of psychotherapy for depression in a low-income country. To date, the few published reports of randomised controlled trials of nonpharmaceutical interventions in such countries have been clinic-based and too

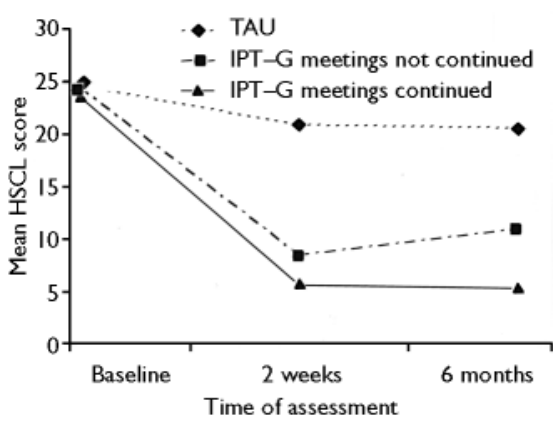

Fig. 2 Depression scores over the study period in intervention recipients who did not attend group meetings during the 6-month follow-up period (group interpersonal psychotherapy (IPT-G) meetings not continued; $n=15$ ), intervention recipients who did attend group meetings during this period (IPT-G meetings continued; $n=88$ ) and the control group receiving treatment as usual (TAU; $n=13$ ). HSCL, Hopkins Symptom Checklist. 
few to form conclusions. A Chilean trial of a psychoeducational group intervention for depressed women at primary care clinics found a significant reduction in depressive symptoms among those treated compared with women receiving usual care at both 3-month and 6-month post-intervention follow-ups (Araya et al, 2003). In contrast, results from the general psychological treatment arm of an intervention trial, consisting of relaxation exercises and symptomfocused problem-solving, for depressed adults attending a medical out-patient clinic in India, reported no short-term or long-term treatment benefit compared with placebo treatment (Patel et al, 2003). Although Patel and colleagues did not find that the psychological intervention was more efficacious than placebo treatment, they did find that the initial reductions in symptom scores for both treatments were generally maintained over time at both the 6-month and 1-year follow-up assessments (Patel et al, 2003). These results are in concordance with the sustained effects we found in our 6-month follow-up.

\section{Choice of intervention strategy}

This research was done to assist World Vision to identify locally appropriate and effective interventions for depression. In Masaka and Rakai, as in other parts of sub-Saharan Africa, non-governmental organisations are the major providers of mental health services where such services exist. Outside the Ugandan capital city of Kampala, the major alternative is traditional healers (Ndyanabangi et al, 2004), but conversations with local healers suggested that they did not believe they had much to offer for depression-like illnesses. Thus, although it might be beneficial in some parts of the world to work with the primary healthcare system so that the intervention could be scaled up and sustained nationally, in regions where the infrastructure is not yet in place to do this, working with well-established non-governmental organisations is a good alternative.

The decision to employ psychotherapy was made with World Vision on the basis of expense and infrastructural limits. Treating depression with psychoactive medication in this population was determined to be impractical for both World Vision and local government, given the cost of antidepressants, the cost of employing medically trained personnel to monitor dosage and side-effects, and the potential logistical problems in maintaining the supply of the drugs in the region. Interpersonal psychotherapy in a group format was selected specifically because it was considered the most culturally appropriate intervention for this population, based on results of our previous qualitative research (Bolton et al, 2004) and on discussions with representatives of the local population. Its focus on relationships as a basis for problem-solving leading to reduction in depressive symptoms was judged to be more in accord with local cultural practice than, for example, the individual, cognitive focus of cognitive-behavioural therapy (Weissman et al, 2000). In addition, interpersonal therapy has been shown to be effective for treating depressive symptoms in HIV-positive patients (Markowitz et al, 1998), which is important given the high prevalence of HIV infection in this region of Africa. The population for this study lived in south-west Uganda, a region severely affected by the HIV epidemic, with prevalence rates estimated at $16.1 \%$ and with HIV/AIDS accounting for nearly two-thirds of adult deaths in the area (Sewankambo et al, 2000).

A post hoc analysis of a possible doseresponse relationship between number of intervention sessions attended and severity of subsequent depression found that the more sessions attended the lower the overall depression score, although this effect was not statistically significant, potentially because of the lack of power to detect the comparatively small effect produced.

\section{Limitations}

We compared group interpersonal psychotherapy with treatment as usual, although we did not systematically evaluate what other treatments people actually sought, if any. We chose treatment as usual as the control condition because our primary purpose was to determine whether the addition of this intervention would be a useful activity for our collaborating partner to undertake. A problem with this approach is that we cannot make a systematic comparison of group interpersonal psychotherapy with other forms of treatment available in the community. To explore this issue we conducted a short, open-ended survey at the follow-up assessment on the self-reported use of counselling services in the 6 months since the intervention ended. Sixty per cent of participants in the intervention group but only
$16 \%$ of the control group reported seeking some sort of counselling. The definition of counselling was left purposely vague, to capture multiple types of services. The type of counselling mentioned by the control group ranged from asking local development agencies for financial assistance, going to friends and family for emotional support, relying on church and community groups for social support, and getting HIV counselling from a local AIDS organisation. The participants in the intervention group mentioned the same sources but also named other group members as providers of counselling and support. These results suggest that the intervention might have been effective partly through enabling the treatment recipients to access additional social support mechanisms that were helpful in maintaining their non-depressed status. Given the lack of information on the specific treatment accessed by those in the control group, we can conclude only that groups organised for the provision of interpersonal psychotherapy were more efficacious at reducing the burden of depression than allowing study participants to access the locally available services they would normally seek out. Without a control group that met regularly, we cannot disentangle the effect of interpersonal psychotherapy from the effect of meeting as a group on a regular basis. In this regard, we cannot be certain that the observed benefits of the intervention derived specifically from the provision of the therapy per $s e$. We plan to investigate this in future studies. At present we can only say that membership of an interpersonal psychotherapy group was more effective in reducing depression than not being part of such a group.

The interviewers were staff members of World Vision and as such worked in the general geographical area of the intervention trial. We attempted to mask the interviewers to the participants' treatment status by not revealing participants' treatment assignments or villages of origin. Because many of the interviewers were familiar with the study villages and might have personally worked with some of the study participants over the course of the trial (in their roles as World Vision staff), the extent to which masking was successfully achieved is not known. We do not know of anything that the interviewers might gain by deliberately biasing the results, because they were not involved in the provision of the treatment and would not personally benefit if 
the treatment were found to be more or less efficacious.

We did not directly ascertain the HIV status of the study participants and thus cannot distinguish between depression as a secondary effect of HIV/AIDS and major depressive disorder as specified in DSM-IV. However, the theoretical perspective of interpersonal psychotherapy is that it sees the depressive episode as nested in perturbed human relationships or fuelled by stressful life events, and since a diagnosis of AIDS and all the social and practical turmoil that follows represent stressful events, depression secondary to AIDS is entirely consistent with the therapeutic model.

\section{Implications of our findings}

Achieving long-term sustained benefits of mental healthcare is particularly important in low-income countries, where scarcity of resources virtually precludes frequent offerings of further interventions. These results are impressive, considering that our trainers had not worked in Africa previously and that this was the first time that the group leaders had had any mental health training or been involved in an intervention trial. Under these circumstances the initial intervention effects, together with the sustainability of these effects over 6 months, speak to the potential feasibility and effectiveness of these types of interventions in the future. No further assessment was conducted on this sample, in part because, following the strong results presented here, World Vision began to provide the therapy to those in the control group still requiring assistance.

The results from this trial indicate that the intervention effects seen immediately after the completion of the intervention were sustained 6 months later. Whether the results represent a direct effect of the 16-week intervention or whether they represent an indirect benefit of the intervention in creating social network groups that continued to meet after the formal intervention was complete is still up for investigation. Regardless of which explanation is correct, group interpersonal psychotherapy as tested here appears to be both feasible and effective.

\section{ACKNOWLEDGEMENTS}

The Mellon Foundation funded this project but had no role in data collection, analysis, manuscript

\section{CLINICAL IMPLICATIONS}

Providing a 16 -week course of group interpersonal psychotherapy produced long-term benefits in reducing depressive symptoms among rural Ugandans.

- The initial reductions in social and physical dysfunction associated with the improved depression symptoms were maintained over the follow-up period.

- The intervention strategy was well accepted by the community, as evidenced by the continuation of informal group meetings once the formal intervention was completed.

\section{LIMITATIONS}

There was limited information on non-study treatment and services used by intervention and control participants.

- The sample size was too small to investigate variations in intervention efficacy by HIV/AIDS status and other health conditions.

- The continuation of informal group meetings made it difficult to evaluate the long-term effect of the specific time-limited therapy independent of follow-up period.

JUDITH BASS, PhD, MPH, Applied Mental Health Research Group (AMHR), Center for International Health and Development, Boston University School of Public Health, Boston, Massachusetts; RICHARD NEUGEBAUER, Epidemiology of Developmental Brain Disorders Department, New York State Psychiatric Institute, G. H. Sergievsky Center, Faculty of Medicine, College of Physicians and Surgeons, Columbia University, New York, and Department of International Health, School of Public Health and Tropical Medicine, Tulane University, New Orleans, Louisiana; KATHLEEN F. CLOUGHERTY, HELEN VERDELI, PRIYA

WICKRAMARATNE, Division of Clinical and Genetic Epidemiology, New York State Psychiatric Institute and Department of Psychiatry, Columbia University College of Physicians and Surgeons, New York; LINCOLN NDOGONI, World Vision International, Washington, DC, USA; LIESBETH SPEELMAN, War Child Netherlands, Amsterdam, The Netherlands; MYRNA WEISSMAN, Division of Clinical and Genetic Epidemiology, New York State Psychiatric Institute and Department of Psychiatry, Columbia University College of Physicians and Surgeons, New York; PAUL BOLTON, AMHR, Center for International Health and Development, Boston University School of Public Health, Boston, Massachusetts, USA

Correspondence: Dr Judith Bass, Center for International Health and Development, Boston University School of Public Health, 85 East Concord Street, 5th Floor, Boston, MA 021I8, USA. Tel: + 617414 1264; fax: + 617414 1261; e-mail: jbass@bu.edu

(First received 14 October 2004, final revision 25 July 2005, accepted 23 August 2005)

preparation or authorisation for publication. R.N.'s efforts on this project were supported in part by a grant from the Ruth and David Levine Foundation and the International Program on Refugee Trauma, of which R.N. is the director. Material support was provided by our collaborator World Vision International in the form of vehicles, drivers and local staff time. We thank the staff, local leaders and communities who welcomed us to work with them.

\section{REFERENCES}

American Psychiatric Association (1994) Diagnostic and Statistical Manual of Mental Disorders (4th edn) (DSM-IV). Washington, DC: APA.
Araya, P., Rojas, G., Fritsch, R., et al (2003) Treating depression in primary care in low-income women in Santiago, Chile: a randomized controlled trial. Lancet, 361, 995-100.

Bolton, P. (200I) Cross-cultural validity and reliability testing of a standard psychiatric instrument without a gold standard. Journal of Nervous and Mental Disease, 189, 238-242.

Bolton, P. \& Tang, A. M. (2002) An alternative approach to cross-cultural function assessment. Social Psychiatry and Psychiatric Epidemiology, 37, 537-543.

Bolton, P., Neugebauer, R. \& Ndogoni, L. (2002) Prevalence of depression in rural Rwanda based on symptom and functional criteria. Journal of Nervous and Mental Disease, 190, 631-637.

Bolton, P., Bass, J., Neugebauer, R., et al (2003) Group interpersonal psychotherapy for depression in 
rural Uganda: a randomized controlled trial. JAMA, 289 , 3117-3124.

\section{Bolton, P., Wilk, C. I. \& Ndogoni, L. (2004)}

Assessment of depression prevalence in rural Uganda using symptom and functional criteria. Social Psychiatry and Psychiatric Epidemiology, 39, 442-447.

Demyttenaere, K., Bruffaerts, R., Posada-Villa, J., et al (2004) Prevalence, severity, and unmet need for treatment of mental disorders in the World Health Organization World Mental Health Surveys. JAMA, 29I, $2581-2590$.

Derogatis, L. R., Lipman, R. S., Rickels, K., et al (1974) The Hopkins Symptom Checklist (HSCL): a selfreport symptom inventory. Behavioral Science, 19, I-15.

Jané-Llopis, E., Hosman, C., Jenkins, R., et al (2003) Predictors of efficacy in depression prevention programmes: meta-analysis. British Journal of Psychiatry, 183, 384-397.

Markowitz, J. C., Kocsis, J. H., Fishman, B., et al (1998) Treatment of depressive symptoms in human immunodeficiency virus-positive patients. Archives of General Psychiatry, 55, 452-457.

Mollica, R. F., Wysak, G., de Marneffe, D., et al (1987) Indochinese versions of the Hopkins Symptom
Checklist-25: a screening instrument for the psychiatric care of refugees. American Journal of Psychiatry, 144 497-500.

Mollica, R. F., Mclnnes, K., Sarajlic, N., et al (1999) Disability associated with psychiatric comorbidity and health status in Bosnian refugees living in Croatia. JAMA, 28, 433-439.

Mulder, R.T., Joyce, P. R., Frampton, C. (2003) Relationships among measures of treatment outcome in depressed patients. Journal of Affective Disorders, 76 127-135.

Ndyanabangi, S., Basangwa, D., Lutakome, J., et al (2004) Uganda mental health profile. International Review of Psychiatry, 16, 54-62.

Patel, V. (1998) Culture and Common Mental Disorders in Sub-Saharan Africa: Studies in Primary Care in Zimbabwe. Hove: Psychology Press.

Patel, V., Chrisholm, D., Rabe-Hesketh, S., et al (2003) Efficacy and cost-effectiveness of drug and psychological treatments for common mental disorders in general health care in Goa, India: a randomized, controlled trial. Lancet, 36I, 33-39.

Sabin, M., Lopes Cardozo, B., Nackerud, L., et a (2003) Factors associated with poor mental health among Guatemalan refugees living in Mexico 20 years after civil conflict. JAMA, 290, 635-642.

Sewankambo, N. K., Gray, R. H., Ahmad, S., et al (2000) Mortality associated with HIV infection in rural Rakai District, Uganda. AIDS, 14, 239I-2400.

Sumathipala, A., Hewege, S., Hanwella, R., et al (2000) Randomized controlled trial of cognitive behaviour therapy for repeated consultations for medically unexplained complaints: a feasibility study in Sri Lanka. Psychological Medicine, 30, 747-757.

Verdeli, H., Clougherty, K., Bolton, P., et al (2003) Adapting group interpersonal psychotherapy for a developing country: experience in rural Uganda. World Psychiatry, 2, I14-120.

Weissman, M. M., Markowitz, J. \& Klerman, G. L. (2000) Comprehensive Guide to Interpersonal Psychotherapy. New York: Basic Books.

Wilk, C. M. \& Bolton, P. (2002) Local perceptions of the mental health effects of the Uganda acquired immunodeficiency syndrome epidemic. Journal of Nervous and Mental Disease, 190, 394-397.

World Health Organization (200I) Mental Health: New Understanding, New Hope. Geneva: WHO. 